\title{
VLSI Implementation of IDMA based Multiple Carrier Code Division Multiple Access for 4G Technologies
}

\author{
K.Veena Devi \\ M.E/Applied Electronics- II Year \\ Tagore Engg College \\ Chennai
}

\author{
P.Radhakrishnan \\ Sr.Asst.Prof/ECE \\ Tagore Engg College \\ Chennai
}

\author{
S.Prakash, PhD. \\ Prof/ECE \\ Jerusalem College of Engg \\ Chennai
}

\begin{abstract}
The aim of this paper is to design a transceiver that achieves a data rate of minimum $4 \mathrm{Gbps}$ equivalent to the $4 \mathrm{G}$ standard of IEEE. Multiple Carrier Code Division Multiple Access (MCCDMA) is the combination of code division multiple access (CDMA) and orthogonal Frequency Division Multiplexing (OFDM), therefore the advantages of both schemes can be achieved. In MC-CDMA scheme each data symbol is spreaded in frequency domain and transmitted on different subcarrier which eliminates frequency selective fading and show significant improvement in bit error rate performance. The existing system uses 1-dimensional interleaver for storing data from spreader. It has insufficient memory to interleave incoming data that provides bottleneck that reduces total system efficiency. To overcome this problem, a method called reconfigurable interleaver division multiple access (IDMA) based MCCDMA is proposed. It provides array type of interleaver that has multiple rows and multiple columns, so it can adapt to changes array based on incoming data. Thus it has efficiency to handle bottleneck by processing multiple data. Thus the throughput of the system will be increased.
\end{abstract}

\section{Keywords}

\section{CDMA; IDMA; MC-CDMA; OFDM}

\section{INTRODUCTION}

MC-CDMA is a promising scheme because of an efficient fast Fourier transform (FFT) technique and multiple access technique. It spreads the multiuser data signal in narrow band spectrum which can reduce effect of multi path frequencyselective fading channel and prevents inter symbol interference (1SI)[4]. MC-CDMA is a technique for overcoming the relative capacity limitation of direct-sequence CDMA (DS-CDMA) and now developed for high-data-rate wireless communication standards[11]. Multiple - input multiple-output (MIMO) array antenna schemes are an interesting idea to fulfill the requirements on bandwidth efficiency and reduce effect of fading channel which these techniques can usefully move the diversity from the base stations (BS) to the mobile units[5].

\section{FREQUENCY INTERLEAVING}

Interleaver simply generates permuted sequence of input data elements. Associated with any interleaver is a de-interleaver that restores the original input data sequence. Interleavers are generally used after channel coding so as to rearrange the ordering of coded data. The channel coding techniques are designed to detect and correct a specific number of random errors depending on the error detecting and correcting capability of the given code. The performances of random error correcting codes are worst in fading channel because they cannot handle long burst of error. Moreover, burst error correcting codes are computationally expensive to correct long burst of error. Hence, in order to make it possible to use simple random error correcting codes, in typical wireless communication system, interleaver is employed after channel coding. The purpose to introduce interleaver after channel coding is to improve the performance of channel coding.

\section{PROBLEM STATEMENT CONTRIBUTION}

In recent years a new multiple access technique, where the users are separated through their unique interleaving patterns, has generated a large interest in the research community. The technique, referred to as interleave-division multiple access (IDMA), has been shown to mitigate multiple access interference while simultaneously achieving a high spectral efficiency [6]. The performance of code-division multipleaccess (CDMA) systems is mainly limited by multiple access interference (MAI) and Inter Symbol Interference (ISI).

A conventional random waveform CDMA (RW-CDMA) system (such as IS-95) involves separate coding and spreading operations. Theoretical analysis shows that the optimal multiple access channel (MAC) capacity is achievable

when the entire bandwidth expansion is devoted to coding. This suggests combining coding and spreading using low rate codes to maximize the coding gain. In this case, interleavers can be employed to distinguish signals from different users and low receiver complexity. This scheme relies on interleaving as the only means to distinguish the signals from different users, and hence it has been called interleave division multiple-access (IDMA). IDMA inherits many advantages from CDMA, in particular, diversity against fading and mitigation of the worst-case other cell user interference problem. Furthermore, it allows a very simple chip-by-chip iterative Multi user Detection (MUD) strategy[1]. The normalized MUD cost (per user) is independent of the number of users.

In this paper throughput performance of MCCDMA over IDMA is investigated. A comparison is made between 1-D interleaver in MCCDMA and MCCDMA over IDMA among the different number of users. Simulation results shows that the throughput increases in IDMA based MCCDMA technique with number of users. 
The rest of the paper is organized as follows: Section 4 describes the transmitter and receiver model for MCCDMA system. Section5 focuses on interleaver design. Performance results are shown in section7 and conclusion is given in section8.

\section{SYSTEM MODEL}

Fig-1 shows the simulation model of MC-CDMA transmitter. Randomly generated user data are randomized and output of randomizer is fed to forward error correction (FEC) block where randomized data are coded by Reed
Solomon and convolutional encoder. FEC code has high gain and adopted in WCDMA and WiMAX standards[10]. Viterbi algorithm is used for decoding the convolutional coding[9]. Output of FEC code is interleaved and modulated[12]. For modulation 16-QAM is used. Modulated output is spreaded by Walsh - Hadamard spreading code in frequency domain then transmitted simultaneously on $N c$ parallel subcarriers[8]. Spreading will convert narrowband signal to wideband signal to suppress the self-interference by multipath effect. Various types of spreading codes, like Walsh- Hadamard (W-H) code, Pseudo Noise (PN) spreading code,

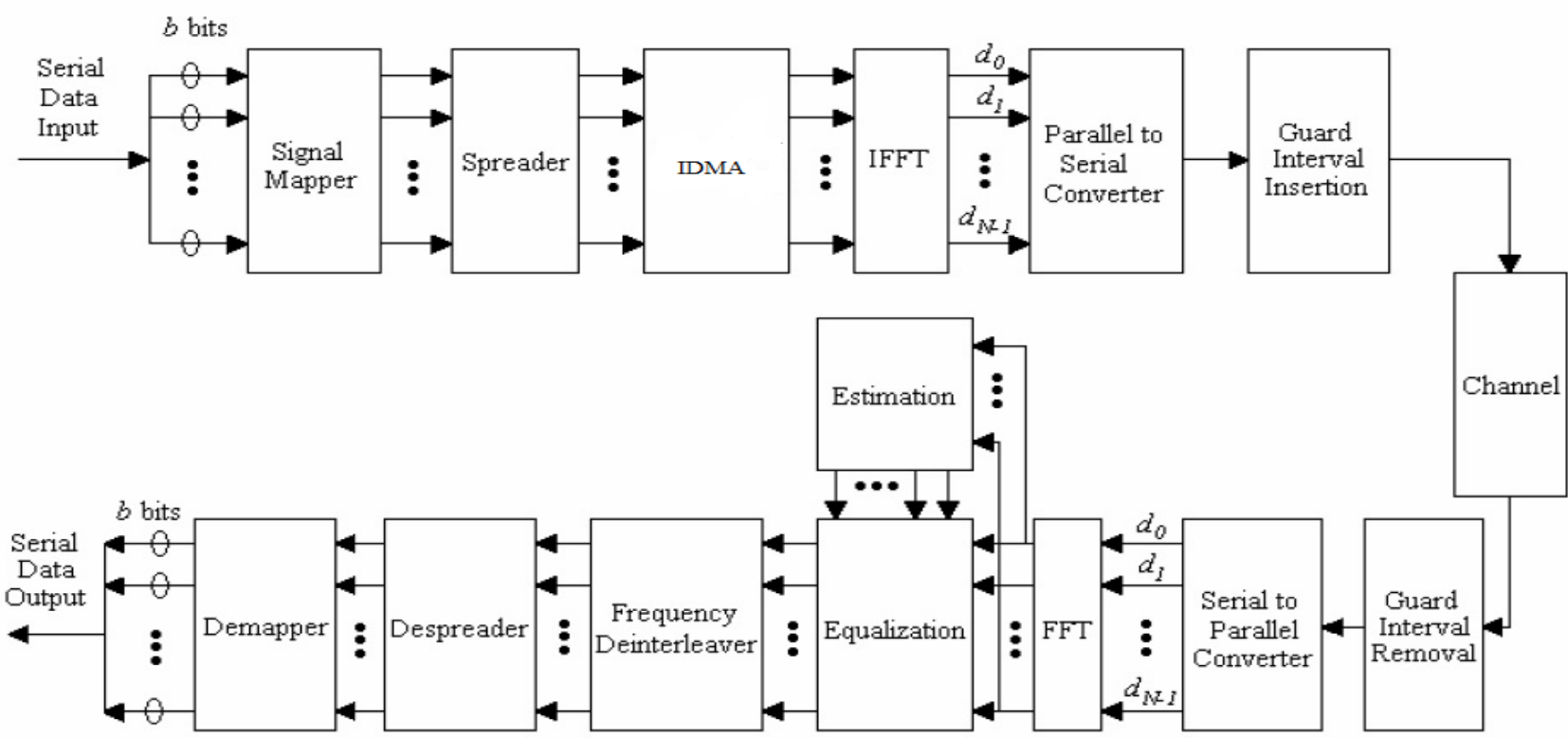

Fig 1: IDMA based MCCDMA model

Gold code, Golay code are used to spread the user data. These codes can be distinguished each other from their correlation properties, orthogonality and peak average to power ratio (PAPR). Since W-H code is orthogonal code its performance is good among the above spreading code. To minimize the MAI spreading code should be orthogonal. In synchronous downlink for MAI reduction W-H code can be used. In uplink PN code is used because due to distortion spreading codes orthogonality gets lost. Also PN sequences are employed in FHSS system[2]. In view of correlation property Gold code is suitable option. For asynchronous transmission W-H code and Gold code are used in WCDMA standard. Signals become more sensitive to non linearities of transmitter for high peak average to power ratio. In uplink PAPR can be reduced by Golay code. These parallel subcarriers are orthogonal to each other and can be generated by using Inverse Fast Fourier transform (IFFT). After this cyclic prefix is used as a guard interval to minimize the effect of inter carrier interference (ICI). Now parallel to serial converter (P/S) converts parallel data into serial data stream and transmit over channel. At receiver first convert serial data into parallel by serial to parallel converter $(\mathrm{S} / \mathrm{P})$ then remove the cyclic prefix and take the Fast Fourier Transform (FFT) of received signal. After that despread and demodulate the users signal. The output of demodulator passes through the channel decoder. The decoded output is derandomized and obtained the users data.

\section{INTERLEAVER}

The input data sequence $\boldsymbol{b}_{\mathrm{n}}$ of user-n is encoded based on a low-rate code $\mathrm{C}$, generating a coded sequence $\boldsymbol{c}_{\mathrm{k}}=$ $\left[c_{k}(1), \ldots, c_{k}(j), \ldots, c_{k}(J)\right]^{T}$, where $J$ is the frame length. The elements in $\boldsymbol{c}_{\mathrm{k}}$ are referred to as coded bits. Then $\boldsymbol{c}_{\mathrm{k}}$ is permutated by an interleaver $=\mathrm{k}$, producing $\boldsymbol{x}_{\mathrm{k}}=\left[\mathrm{x}_{\mathrm{k}}(1), \ldots\right.$, $\left.\mathrm{x}_{\mathrm{k}}(\mathrm{j}), \ldots, \mathrm{x}_{\mathrm{k}}(\mathrm{J})\right]^{\mathrm{T}}$. The elements in $\boldsymbol{x}_{\mathrm{k}}$ "chips". Users are solely distinguished by their interleavers, hence the name interleavedivision multiple-access(IDMA). The key principle of IDMA is that the interleavers $\{=\mathrm{k}\}$ should be different for different users.It is assumed that the interleavers are generated independently and randomly. These interleavers disperse the coded sequences so that the adjacent chips are approximately uncorrelated, which facilitates the simple chip-by-chip detection scheme.

$$
\mathrm{D}_{\mathrm{R}}=\mathrm{F}_{\mathrm{R}} * \mathrm{~N}_{\mathrm{T}}
$$

Where $D_{R}$ is the data rate of the active user, $F$ is the frequency of the transmitter or receiver, $N_{T}$ is the number of bits transmitted.

\section{CHANNEL ESTIMATION USING THE FFT METHOD}

A Rayleigh fading compensation technique using the FFT method was originally proposed for QAM signals[3]. The same technique is applied for a MC-CDMA signal. Pilot chips are inserted before the IFFT block at the transmitter or can be said to be in the frequency domain. The least squares 
estimate of the pilots is obtained in the receiver by dividing those corrupted pilots with the known pilot symbols. To obtain the channel coefficients for the data symbols interpolation is performed in the frequency domain In conventional CDMA, only one carrier signal is modulated and thus if the signal undergoes any multipath fading, then the total signal may be distorted. Multiple carrier modulation technique like MC-CDMA allows the receiver to receive information correctly even when some of subcarriers are affected by multipath fading.

\section{RESULTS \& DISCUSSIONS}

Each block of MC-CDMA transmitter and receiver is individually coded. Reed Solomen and convolution code is used as a channel coding. W-H code has been chosen for spreading the user data, cyclic prefix is taken as $1 / 4$, number of subcarrier $N c$ is kept equal to the spreading code length. The transmitted data rate for 32 data bits at the frequency of $130.4 \mathrm{MHz}$ is $4.17 \mathrm{Gbps}$. The receiver data rate at the frequency of $194.14 \mathrm{MHz}$ is $6.21 \mathrm{Gbps}$. The total data rate is the average of transmitter and receiver data rates. For an ordinary interleaver the throughput achieved is $1.5 \mathrm{Gbps}$ and with IDMA the throughput achieved will be minimum of $4 \mathrm{Gbps}$.For an ordinary interleaver $325 \mathrm{MHz}$ of frequency is achieved whereas in reconfigurable interleaver executes at speed of $1684 \mathrm{MHz}$ frequency.

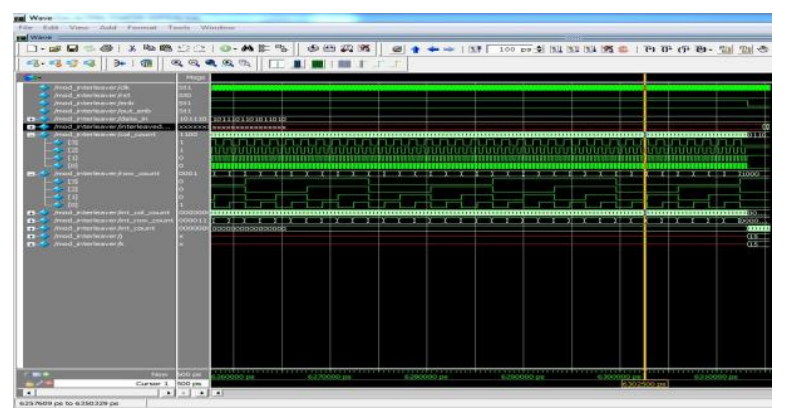

Fig 2: Simulation results of IDMA

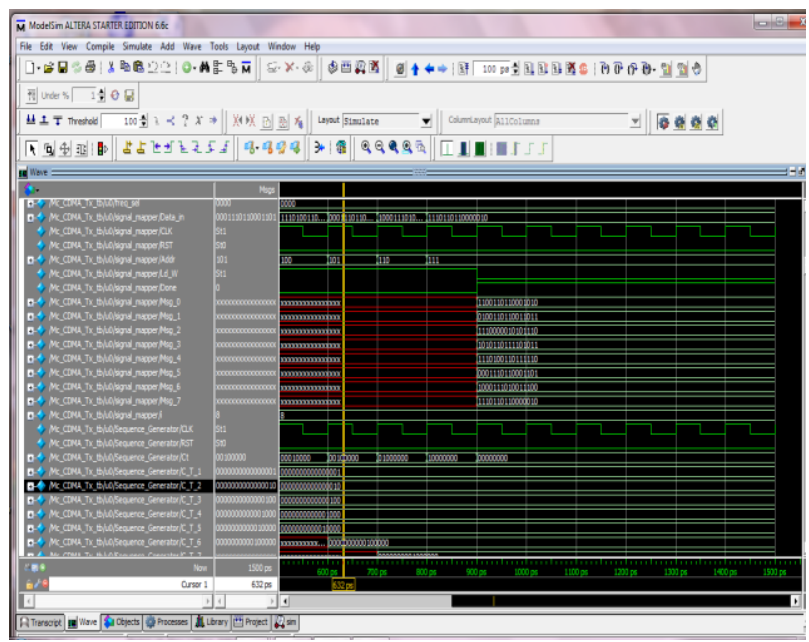

Fig 3: IDMA-MCCDMA Transmitter module

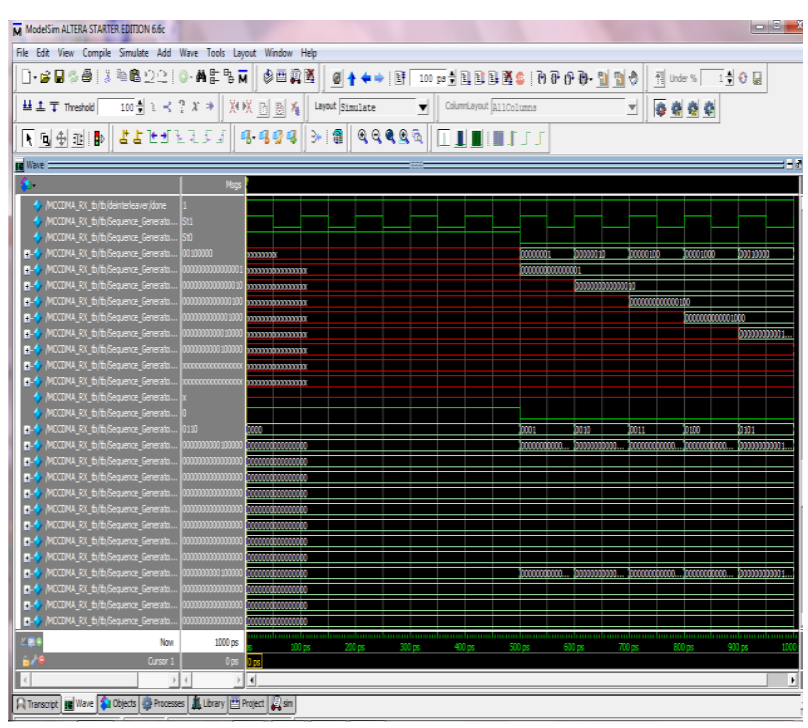

Fig 4: IDMA-MCCDMA Receiver module

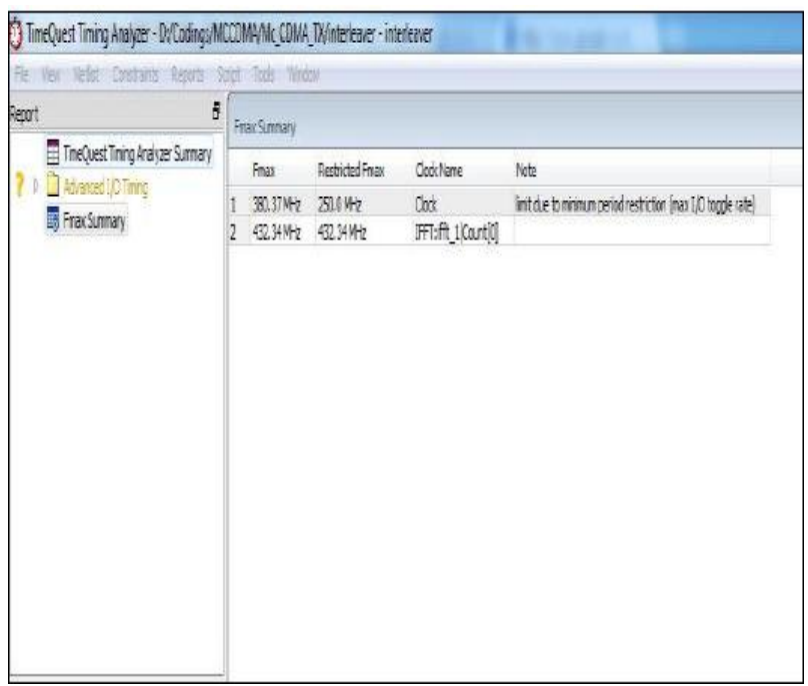

Fig 5: Throughput result of 1-Dimensional interleaver

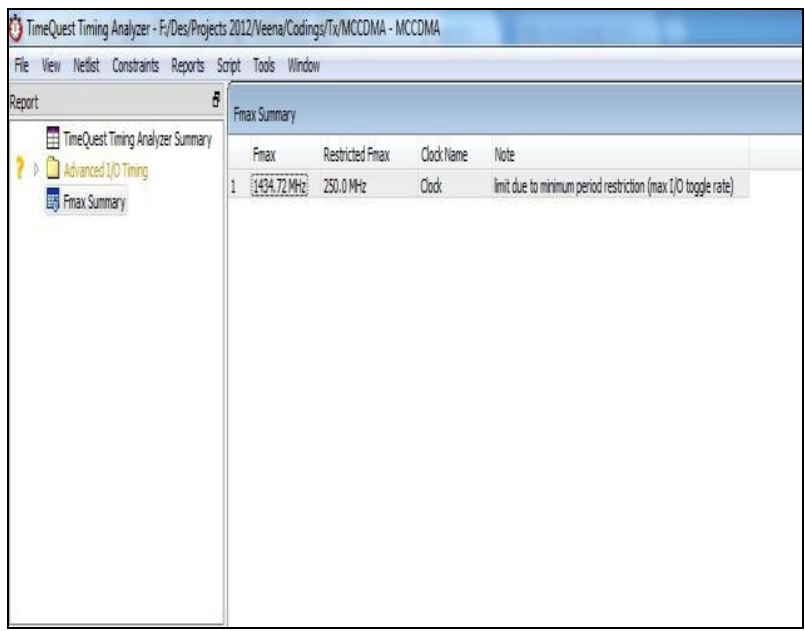

Fig 6: Throughput result of IDMA 


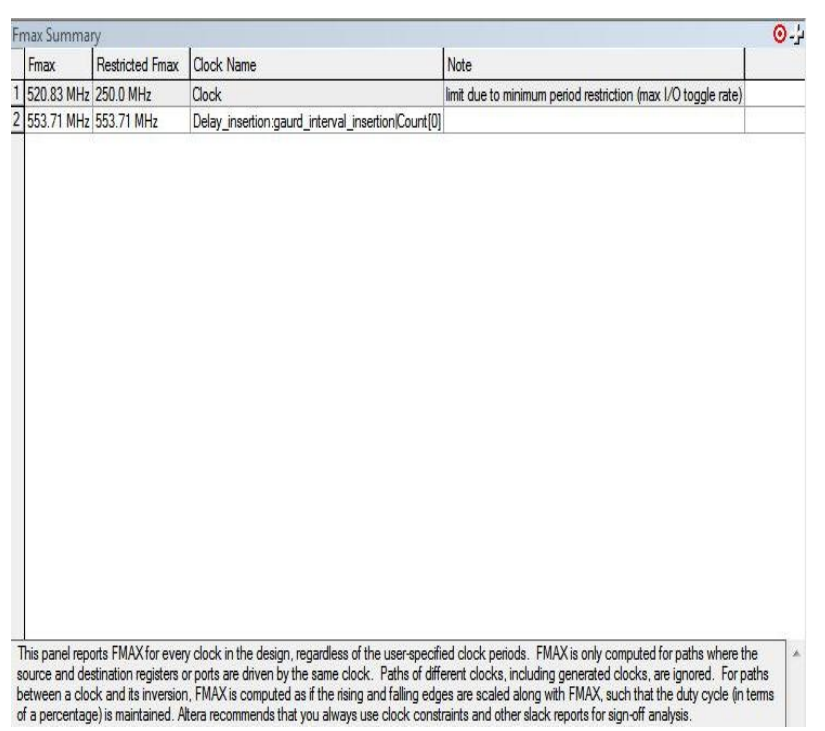

Fig 7: Transmitter Throughput

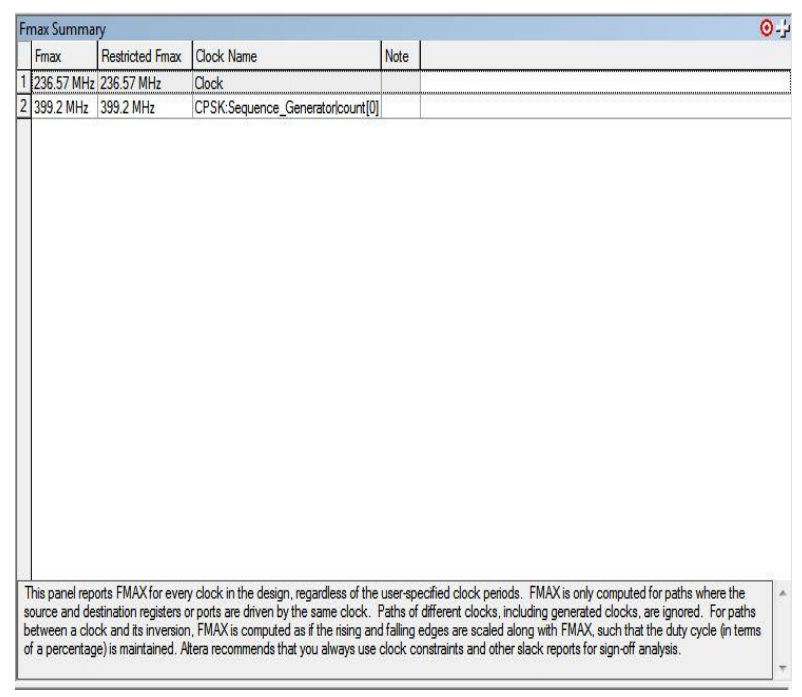

Fig 8: Receiver throughput

Table 1. Comparison between 1 - D based MC -CDMA \&

IDMA based MC - CDMA

\begin{tabular}{|c|c|c|}
\hline $\begin{array}{c}\text { Syatem } \\
\text { Name }\end{array}$ & $\begin{array}{c}\text { Interleaver } \\
\text { capacity }\end{array}$ & $\begin{array}{c}\text { Transmitter } \\
\text { Capacity }\end{array}$ \\
\hline $\begin{array}{c}\text { 1-D interleaver } \\
\text { based MC - CDMA }\end{array}$ & 6.085 Gbps & 2.5 Gbps \\
\hline IDMA based & 22.955 Gbps & $8.33 \mathrm{Gbps}$ \\
MC - CDMA & & \\
\hline
\end{tabular}

\section{CONCLUSIONS}

In this paper throughput performance of MCCDMA over IDMA is described. Improved data rate performance will increase the capacity of the system. The simulation result shows that the throughput is high enough to support $4 \mathrm{G}$ technologies. When Compare with the 1D interleaver in MCCDMA, the IDMA based MCCDMA produce high data rate for the same number of data. In further the simulation results for IDMA based MCCDMA and comparisons between 1-D interleaver based MCCDMA and IDMA based MCCDMA to were done. In future work channel equalization techniques can be obtained for receiver in this method.

\section{REFERENCES}

[1] A.Muayyadi,M.N.A.AbuRgheff,"Wavelet-based multicarrier CDMA system and its corresponding multiuser detection", IEE Proc.-Commun., Vol. 150, No. 6,2003 .

[2] Chenghai Zhang, Jianhao Hu, "The Shifting Interleaver Design Based onPN Sequence for IDMA systems," 2007 International Conference on Future Generation Communication and Networking ,FGCN2007, Korea,Dec, 2007."

[3] D. N. Kalofonos, M. Stojanovic, and J. G. Proakis, "Performance of a MC-CDMA system in a Rayleigh fading channel, in the presence of channel estimation errors," in Proc. 6th Int. Conf. Advances in Communications and Control (COMCON 6), pp. 729 737' June 1997,

[4] Hara,S. and Prasad, R., "Overview of Multicarrier CDMA", in IEEE Comm. Magazine, vol. 35, no. 11, Dec. 1997.

[5] G.J.Foschini,"Layered space-time architecture for wireless communication in a fading environment when using multielement antennas," Bell Labs Tech. J., pp. 41-59, 1996.

[6] Li Ping, Lihai Liu, Keying $\mathrm{Wu}$ and W.K.Leung. "Interleave-DivisionMultiple-Access".IEEE Transactions on Wireless Communication,VOL.5, NO.4,PP.938-947, April 2006.

[7] N. Saad, Abdul-Majeed, "A multiwavelet based MCCDMA system", Ph.D. thesis, College of Engineering,Bagdad University, 2006.

[8] S.-H. Tsai, Y.-P. Lin, and C.-C. J. Kuo, "MAI-free MCCDMA systems based on Hadamard-Walsh codes," IEEE Trans. Signal Processing,vol. 54, no. 8, pp. 31663179, Aug. 2006.

[9] V. D. Nguyen and H. Kuchenbecker, "Block interleaving for soft decision viterbi decoding in OFDM systems," in IEEE VTS 54th Vehicular Technology Conference, VTC 2001 Fall, 2001, pp. 470-474,2001.

[10] Clark, George C., Jr.; Cain, J. Bibb, Error-Correction Coding for Digital Communications. New York: Plenum Press. ISBN 0-306-40615-2, 1981.

[11] Yee N., Linnartz J-P and Fettwis G.,"Multicarrier CDMA in indoor wireless radio networks",Proc. of IEEE PIMRC, Yokohama, Japan, pp. 109-113, 1993.

[12] Ming-Fong Tsai, Ce-Kuen Shieh, Senior Member, IEEE, Tzu-Chi Huang, and Der-Jiunn Deng, Member, IEEE, "Forward-Looking Forward Error Correction Mechanism for Video Streaming over Wireless Networks", IEEE SYSTEMSJOURNAL,DOI:10.1109/JSYST.2011.21655 95,2011. 